\title{
Clinicopathological Characteristics and Survival of Triple-Negative Breast Cancer Patients: A Single Institution Study from Egypt
}

\author{
Wael S. Makar
}

\author{
Kasr Al-Ainy Center of Clinical Oncology and Nuclear Medicine (NEMROCK), Kasr Al-Ainy School \\ of Medicine, Cairo University, Cairo, Egypt
}

Background: Triple-negative breast cancer (TNBC) is a subtype of breast cancer that is characterized by being more
aggressive, presentation in younger age, and higher response rate to chemotherapy. It is more likely to recur and to
metastasize early. Methods: Retrospective review of the medical records of TNBC patients treated in a single Egyptian cancer center during a 4-year period.

Results: Sixty-five patients were included. Forty-six percent of patients were $<50$ years old and $22 \%$ had a positive family history. Ten (15\%) patients were metastatic at presentation. Modified radical mastectomy was performed in $42 \%$ of patients and adjuvant chemotherapy was administered in 55\% of them. Relapse occurred in 12 (22\%) out of 55 patients who had no distant metastasis at presentation (M0). The overall survival rate of M0 patients at 12, 24 and 36 months was $96 \%, 91 \%$ and $88 \%$; respectively. The disease-free survival rate of M0 patients at 12, 24 and 36 months was $90 \%, 81 \%$ and $71 \%$; respectively.

Conclusion: TNBC is an aggressive subtype of breast cancer that occurs at younger age. Frequently, it is of high grade and presents in an advanced stage.

Keywords: Triple-negative breast cancer, Clinicopathological characteristics, Treatment outcome, Egypt Corresponding author: Wael Makar, MD; Kasr Al-Ainy Center of Clinical Oncology and Nuclear Medicine (NEMROCK), Kasr Al-Ainy School of Medicine, Cairo University, Cairo, Egypt; Email: wael_makar@yahoo.com

Submitted: 6-April-2019, Revised: 21-April-2019, Accepted: 27-April-2019, Published online: 3-May-2019

\section{INTRODUCTION}

Triple-negative breast cancer (TNBC) is a subtype of breast cancer that tests negative for estrogen receptors (ER), progesterone receptors (PR) and Her2 protein which means that the proliferation of this type of cancer is not fueled by the estrogen and progesterone hormones or by the Her 2 protein ${ }^{1}$. That is why TNBC does not respond to hormonal therapy or to drugs that target Her2 protein receptors ${ }^{2}$.

Triple-negative breast cancer constitutes about 10$20 \%$ of breast cancers ${ }^{3,4}$. It is more aggressive and has a poorer prognosis than other types of breast cancer as studies have shown that TNBC is likely to metastasize early at presentation and to recur after treatment ${ }^{5}$. It tends to present with a higher grade than other subtypes and usually has a basal-like cellular morphology ${ }^{1}$.

Patients with TNBC are more likely to be $<50$ years of age and about $70 \%$ of them have an inherited BRCA mutation, particularly BRCA1 mutation ${ }^{6}$.

This study describes the clinicopathological characteristics, management, recurrence rate and survival of TNBC patients treated in a single cancer institution in Egypt.

\section{METHODS}

This retrospective study included TNBC patients treated at the Kasr Al-Ainy Center of Clinical Oncology and Nuclear Medicine - Cairo University Hospitals in the period from 2011 to 2014 .

After obtaining the institutional ethical approval, the medical records of breast cancer patients were reviewed.

Adult (> 18 years of age) females with immunohistochemistry (IHC) negative expression of ER, PR and Her2Neu were included in the study. Those who had Her2Neu +1 or +2 expression by IHC were included after being proved to be negative by Fluorescence in-situ hybridization (FISH) result.

A total of 162 TNBC patients were identified. Only $65(40.1 \%)$ of them were included in the study. The remaining patients were excluded because of loss of records, incomplete documentation or loss to follow up.

The data retrieved included: patients' characteristics (age at diagnosis, menopausal status and family history), tumor characteristics (grade, size, nodal involvement and metastases at presentation), treatment received (surgery, chemotherapy and radiotherapy) and recurrence data (type, site and date) if any.

Data were described in terms of mean \pm standard deviation (SD) or frequencies and percentage when appropriate. The mean and median disease-free survival (DFS) and overall survival (OS) were estimated using the Kaplan-Meier method. $\mathrm{P}$ value $<0.05$ was considered statistically significant. Statistical analyses were done using IBM SPSS (Statistical Package for the Social Science) software for Microsoft Windows, release 22 (IBM corp., Armonk, NY, USA). 


\section{RESULTS}

Triple-negative breast cancer represented $10 \%$ of all breast cancer treated at our institution during the study period.

Personal characteristics of the included 65 patients are shown in table 1 . The mean age of patients at diagnosis was 49.2 years $( \pm 13.1)$ and almost half of them were below 50 years old. Positive family history was present in $21.5 \%$ of the patients; all were breast cancer except one patient who gave family history of endometrial carcinoma.

Table 1: Patients' characteristics $(n=65)$

\begin{tabular}{lll}
\hline Variable & No. & \% \\
\hline Age at diagnosis & & \\
\hline$<50$ years & 30 & 46.2 \\
\hline$\geq 50$ years & 35 & 53.8 \\
\hline Family History & & \\
\hline Negative & 51 & 78.5 \\
\hline Positive & 14 & 21.5 \\
\hline Menopausal status & & \\
\hline Premenopausal & 32 & 49.2 \\
\hline Postmenopausal & 33 & 50.8 \\
\hline
\end{tabular}

Table 2 illustrates tumor characteristics and treatment lines received. The most common histopathological type was infiltrating duct carcinoma and approximately one third of patients had grade III tumor. At presentation, almost half the patients had positive lymph nodes and $15 \%$ had distant metastases.

The percentage of patients who underwent modified radical mastectomy (MRM) or breast conservative surgery (BCS) was 79\%. Adjuvant chemotherapy was administered in $55.4 \%$ of the patients while neoadjuvant chemotherapy was administered in $44.6 \%$ of patients. An anthracycline-based chemotherapy regimen was the most commonly regimen used, being administered in $61.5 \%$ of patients. Adjuvant radiotherapy was given to $71 \%$ of patients.

Relapse occurred in $12(21.8 \%)$ patients out of the 55 who were not metastatic at diagnosis. Bone was the most common site of distant metastasis followed by lung then liver. Only one patient developed brain deposits.

The OS rate of non-metastatic (M0) patients was $96 \%$ at 12 months, $91 \%$ at 24 months and $88 \%$ at 36 months. Their mean OS time was 57.7 months (95\% Confidence Interval $[\mathrm{CI}]: 53.7-61.6)$ and the median OS was not reached. The 5-year OS rate was $88 \%$ (figure 1).

The mean OS of metastatic (M1) patients was 23.2 months (95\% CI: $15.75-60.69)$ and the median OS was 28.4 months (figure 1).

The DFS rate of M0 patients at 12, 24 and 36 months was $90 \%, 81 \%$ and $71 \%$, respectively. The 5year DFS rate was $71 \%$ (figure 2).

The 12-months OS rate of patients who received neoadjuvant chemotherapy was $84 \%$ while it was $97 \%$ for those who received adjuvant chemotherapy $(\mathrm{p}=$ 0.054 ) (figure 3 ).
Table 2: Tumor characteristics and treatment $(n=65$ patients)

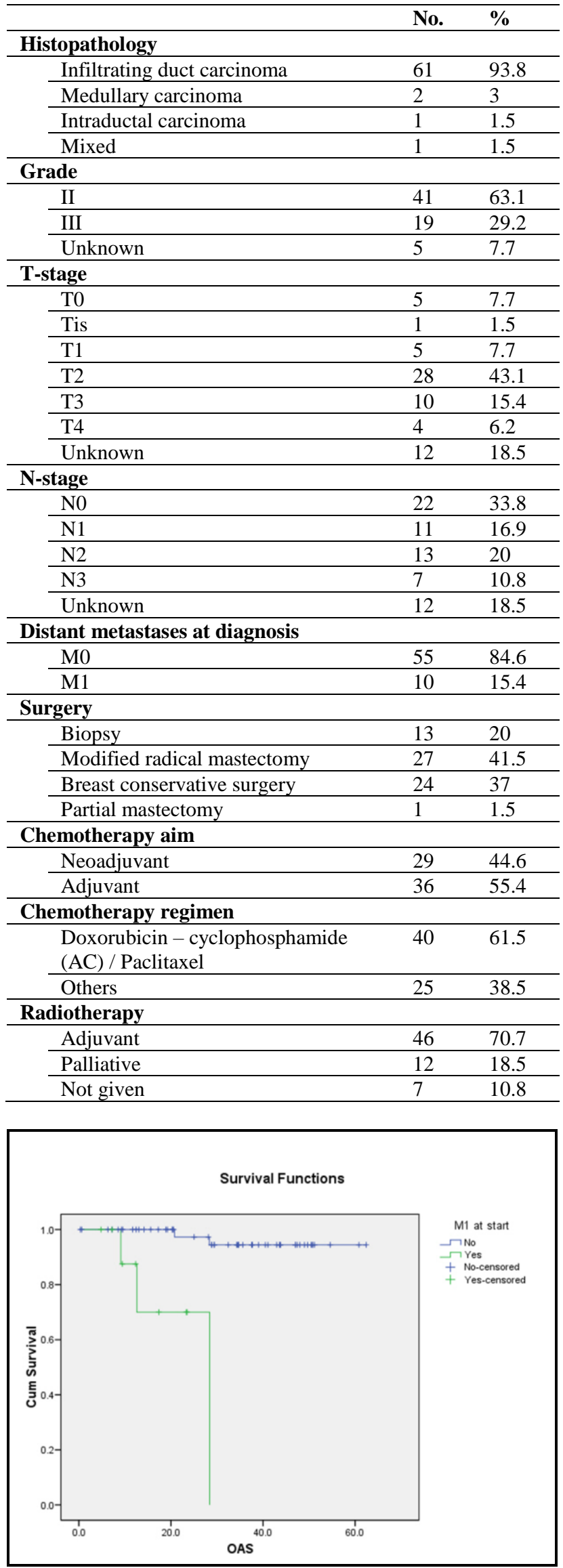

Figure 1: Kaplan-Meier curves of estimated overall survival according to metastatic status at diagnosis 


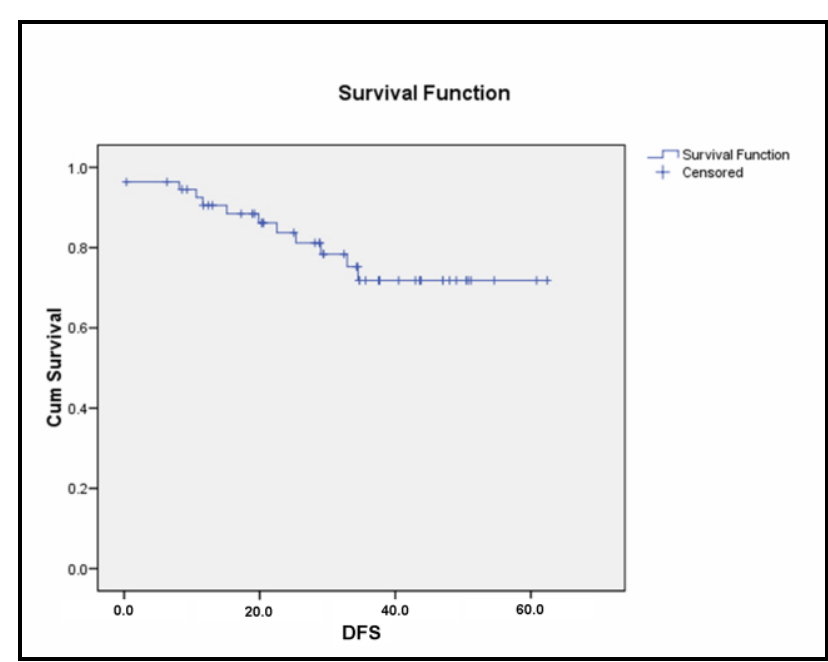

Figure 2: Kaplan-Meier curve of estimated diseasefree survival of M0 patients

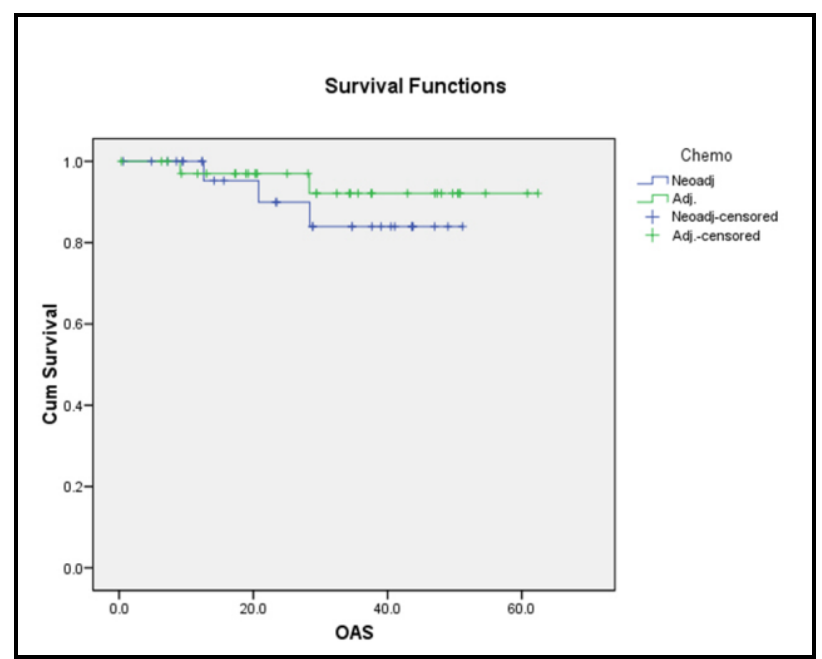

Figure 3: Kaplan-Meier curves of estimated overall survival of patients who received adjuvant vs. neoadjuvant chemotherapy

\section{DISCUSSION}

Triple-negative breast cancer is known by its aggressive clinicopathological features. It presents at a younger age with larger tumor size and higher grade and tends to metastasize and recur after treatment ${ }^{7}$.

At our institution, representing a lower-income setting, TNBC represents $10 \%$ of all breast cancer cases, which is comparable to most of the studies which reported an incidence ranging from $10 \%$ to $20 \%{ }^{4}$.

The mean age of TNBC in our study was 49 years, which is similar to many other studies including those from Egypt where a recent study conducted at the Egyptian National Cancer Institute found a similar mean age of 49 years ${ }^{8}$. However, some other studies reported different mean age ranging from 44 to 56 years 5,7 .

Positive family history was documented in $22 \%$ of our patients similar to that reported by Fayaz et al from Kuwait ${ }^{9}$, while Ghosn et al reported a family history in $10 \%$ of patients with TNBC in Lebanon ${ }^{10}$.

Invasive duct carcinoma is the predominant histopathology type in our study being presented in $94 \%$ of our patients similar to studies done in Singapore [11] and Japan ${ }^{11,12}$.

Most of the cases in our study were T2 stage (43\%) followed by T3 (15\%), and grade II (63\%) followed by grade III $(24 \%)$. This is compatible with the aggressive nature of TNBC which is usually of high grade and large tumor size as reported by other studies ${ }^{13}$.

Zhang et al ${ }^{14}$ and Dent et al ${ }^{15}$ reported a percentage of node positivity among TNBC cases higher than that among our patients. This may be attributed to the small number of patients included in our study. However, Lin et al and other related studies reported that TNBC was less likely to be lymph node positive ${ }^{16}$.

In our study the 1-year OS rate was $84 \%$ for patients who received neoadjuvant chemotherapy, which was lower than the $97 \%$ rate of those who received adjuvant chemotherapy. This could be attributed to the fact that neoadjuvant chemotherapy is administered to patients presenting with more advanced disease to downstage it. However, difference between the two groups was not statistically significant. It is difficult with the current study design and sample size to come out with a conclusion regarding that issue.

The 5-year OS rate in our study was $88 \%$ which is comparable to that reported by a study from China where the 5-year OS rate was $88.5 \%{ }^{17}$, and another study from Iran where the 5 -year survival was $86.1 \%{ }^{18}$. On the other hand, the 5-year OS rates were much lower in other studies. A study from New Zealand reported a 5year OS rate of $72 \%{ }^{19}$ and another study from Brazil done by Gonçalves et al reported a rate of $62 \%{ }^{20}$. This was similar to the $64 \%$ rate found by Liedtke et al ${ }^{21}$. The higher 5-year OS rate in our study could be attributed to the earlier stage (T2 and T3) at presentation among our patients. Studies that reported lower 5-year OS rates included patients with more advanced stage, mostly T3 and $\mathrm{T} 4$ at presentation.

\section{Conclusion}

Triple-negative breast cancer is an aggressive subtype of breast cancer that occurs among younger patients ( $<50$ years old) and usually presents with large tumor size and high grade. This confirms that the clinicopathogical characteristics of TNBC among Egyptian female patients are more or less similar to that in other regions of the World. To improve the treatment outcome of TNBC, novel therapies are needed, such as the PARP inhibitors and antiandrogens. Patients should be encouraged to be treated in large clinical studies that are well-designed using the newly developed molecularly targeted therapy.

\section{REFERENCES}

1- Kaplan HG, Malmgren JA. Impact of triple-negative phenotype on breast cancer prognosis. Breast J. 2008; 14(5): 456-463.

2- Ferlay J, Soerjomataram I, Dikshit R, et al. Cancer incidence and mortality worldwide: sources, methods and major patterns in Globocan 2012. Int J Cancer. 2015; 136(5): E359-386. 
3- Anders CK, Abramson V, Tan T, Dent R. The evolution of triple-negative breast cancer: from biology to novel therapies. Am Soc Clin Oncol Edu Book. 2016; 36: 34-42.

4- Siegel RL, Miller KD, Jemal A. Cancer statistics, 2017. CA Cancer J Clin. 2017; 67(1): 7-30.

5- Foulkers WD, Smith IE, Reis-Filho JS. Triple-negative breast cancer. N Engl J Med. 2010; 363(20): 1938-1948.

6- Robson M, Im SA, Senkus E, et al. Olaparib for metastatic breast cancer in patients with germline BRCA mutation. N Engl J Med. 2017; 377(6): 523-533.

7- Criscitiello C, Azim HA Jr, Schouten PC, Linn SC, Sotiriou C. Understanding the biology of triple-negative breast cancer. Ann Oncol, 2012; 23(Suppl 6): vi13-18.

8- Zakaria A, El-Kinaai N, Loay I, et al. Triple-negative breast cancer, clinicopathological study of Egyptian patients: NCI experience. Med J Cairo Univ. 2018; 86(5): 2747-2753.

9- Fayaz MS, El-Sherify MS, El-Basmy A, et al. Clinicopathological features and prognosis of triplenegative breast cancer in Kuwait: A comparative / perspective analysis. Rep Pract Oncol Radiother. 2013; 19(3): 173-181.

10- Ghosn M, Haji C, Kattan J, et al. Triple-negative breast cancer in Lebanon: a case series. Oncologist. 2011; 16(11): 1552-1556.

11- Park YH, Lee SJ, Cho EY, et al. Clinical Relevance of TNM staging systems according to breast cancer subtypes. Ann Oncol. 2011; 22(7): 1554-1560.

12- Ishikawa Y, Horiguchi J, Toya H, et al. Triple-negative breast cancer: histological subtypes and immunohistochemical and clinicopathological features. Cancer Sci, 2011; 102(3): 656-662.

13- Thike AA, Cheok PY, Jara-Lazaro AR, et al. Triplenegative breast cancer: clinicopathological characteristics and relationship with basal-like breast cancer. Mod Pathol. 2010; 23(1): 123-133.

14- Zhang L, Hao C, Dong G, Tong Z. Analysis of clinical features and outcome of 356 triple-negative breast cancer patients in China. Breast Care (Basel). 2012; 7(1): 13-17.

15- Dent R, Trudeau M, Pritchard KI, et al. Triple-negative breast cancer: Clinical features and pattern of recurrence. Clin Cancer Res. 2007; 13(15 Pt 1): 4429-4434.

16- Lin NU, Vandesplas A, Hughes $M$, et al Clinicopathological features, patterns of recurrence, and survival among women with triple-negative breast cancer in the National Comprehensive Cancer Network. Cancer. 2012; 188(22): 5463-5472.

17- Qiu J, Xue X, Hu C, et al. Comparison of clinicopathological features and prognosis in triplenegative and non-triple negative breast cancer. J Cancer. 2016; 7(2): 167-173.

18- Mousavi SA, Kasaeian A, Pourkasmaee $M$, et al Assessing the prognostic factors, survival, and recurrence incidence of triple negative breast cancer patients, a single center study in Iran. PLoS One. 2019; 14(1): e0208701.

19- James M, Dixit A, Robinson B, Frampton C, Davey V. Outcome for patients with non-metastatic triple-negative breast cancer in New Zealand. Clin Oncol (R Coll Radiol). 2019; 31(1): 17 -24.

20- Gonçalves H Jr, Guerra MR, Duarte Cintra JR, Fayer VA, Brum IV, Bustamante Teixeira MT. Survival study of triple-negative and non-triple-negative breast cancer in a Brazilian cohort. Clin Med Insights Oncol. 2018; 12: 1179554918790563.

21- Liedtke C, Mazouni C, Hess KR, et al. Response to neoadjuvant therapy and long-term survival in patients with triple-negative breast cancer. J Clin Oncol. 2008; 26(8): 1275-1281. 